\title{
Long-Term Safety and Efficacy of Budesonide/
}

\section{Glycopyrrolate/Formoterol Fumarate Metered Dose Inhaler Formulated Using Co-Suspension Delivery Technology in Japanese Patients with COPD}

This article was published in the following Dove Press journal:

International Journal of Chronic Obstructive Pulmonary Disease

\author{
Masakazu Ichinose,' \\ Yasushi Fukushima, (D) ${ }^{2}$ \\ Yoshikazu Inoue, (D) ${ }^{3}$ \\ Osamu Hataji, ${ }^{4}$ Gary T Ferguson, ${ }^{5}$ \\ Klaus F Rabe, ${ }^{6}$ Nobuya Hayashi, $\mathbb{D D}^{7}$ \\ Hiroshi Okada, (ID) ${ }^{7}$ \\ Mami Takikawa, ${ }^{7}$ Eric Bourne, ${ }^{8}$ \\ Shaila Ballal, ${ }^{9}$ \\ Kiernan DeAngelis, (ID) 10 \\ Magnus Aurivillius," \\ Colin Reisner, (1D ${ }^{9}$ \\ Paul Dorinsky $\mathbb{1 D}^{8}$ \\ 'Department of Respiratory Medicine, \\ Tohoku University Graduate School of \\ Medicine, Sendai, Japan; ${ }^{2}$ Department of \\ Internal Medicine, Fukuwa Clinic, Tokyo, \\ Japan; ${ }^{3}$ Clinical Research Center, National \\ Hospital Organization, Kinki-Chuo Chest \\ Medical Center, Osaka, Japan; ${ }^{4}$ Respiratory \\ Center, Matsusaka Municipal Hospital, \\ Matsusaka, Japan; ${ }^{5}$ Pulmonary Research \\ Institute of Southeast Michigan, Farmington \\ Hills, MI, USA; ${ }^{6}$ LungenClinic Grosshansdorf \\ and Christian-Albrechts University Kiel, \\ Airway Research Center North, Member of \\ the German Center for Lung Research \\ (DZL), Großhansdorf, Germany; \\ ${ }^{7}$ AstraZeneca K.K., Osaka, Japan; \\ ${ }^{8}$ AstraZeneca, Durham, NC, USA; \\ ${ }^{9}$ AstraZeneca, Morristown, NJ, USA; \\ ${ }^{10}$ Formerly of AstraZeneca, Durham, NC, \\ USA; " AstraZeneca, Gothenburg, Sweden
}

Correspondence: Masakazu Ichinose

Department of Respiratory Medicine,

Tohoku University Graduate School of

Medicine, Sendai, Japan

$\mathrm{Tel}+8 \mathrm{I}-22-7 \mathrm{I} 7-8534$

Email ichinose@rm.med.tohoku.ac.jp
Background: Budesonide/glycopyrrolate/formoterol fumarate metered dose inhaler (BGF $\mathrm{MDI}$ ) is a triple fixed-dose combination for COPD. The long-term safety of triple therapy for COPD has not been investigated in Japanese patients. In this 28-week extension study (NCT03262012), we investigated the long-term safety and tolerability of BGF MDI in Japanese patients with moderate-to-very severe COPD who completed the 24-week Phase III randomized, double-blind, multicenter KRONOS study (NCT02497001).

Materials and methods: Patients randomized to BGF MDI 320/18/9.6 $\mu$ g, glycopyrrolate/ formoterol fumarate (GFF) MDI 18/9.6 $\mu \mathrm{g}$, budesonide/formoterol fumarate (BFF) MDI 320/ $9.6 \mu \mathrm{g}$, or budesonide/formoterol fumarate dry powder inhaler (BUD/FORM DPI) 400/12 $\mu \mathrm{g}$ twice-daily in KRONOS continued treatment for up to 28 additional weeks. Safety was evaluated over 52 weeks via adverse event (AE) monitoring, electrocardiograms, clinical laboratory testing, and vital sign measurements.

Results: The safety population included 416 patients who received BGF MDI ( $\mathrm{n}=139)$, GFF MDI $(n=138)$, BFF MDI $(n=70)$, or BUD/FORM DPI $(n=69)$. Treatment-emergent AE (TEAE) rates were similar across treatment groups (range: $82.6-82.9 \%$ ). The most frequent TEAEs overall were nasopharyngitis $(32.2 \%)$ and bronchitis $(9.9 \%)$. The incidence of major adverse cardiovascular events was low across groups (range: $0.0-2.9 \%$ ). Over 52 weeks, the incidence of confirmed pneumonia was 9.4\% (BGF MDI), 3.6\% (GFF MDI), 5.7\% (BFF MDI), and $2.9 \%$ (BUD/FORM DPI); in the 28 -week extension period, rates were comparable across groups (range: $2.9-5.7 \%)$. Six deaths were reported (0.7-2.2\% per group); none were considered treatment-related. No clinically meaningful trends were observed in electrocardiograms, laboratory parameters, or vital signs over time in any of the treatment groups.

Conclusion: All treatments were well tolerated over 52 weeks, and the safety profile of BGF MDI was generally comparable to dual long-acting muscarinic antagonist (LAMA)/ long-acting $\beta_{2}$-agonist (LABA) and inhaled corticosteroid (ICS)/LABA therapies. These findings support the long-term tolerability of BGF MDI in Japanese patients with COPD.

Keywords: co-suspension delivery technology, ICS/LAMA/LABA, inhaled corticosteroid, long-acting muscarinic antagonist, long-acting $\beta_{2}$-agonist, Japan

\section{Introduction}

Approximately 5.3 million Japanese adults over the age of 40 have airflow limitation indicative of COPD; however, it is estimated that more than 5 million of these patients are not currently receiving treatment intervention. ${ }^{1}$ Effective treatment of 
COPD can improve lung function, decrease symptoms, and prevent exacerbations, and typically includes bronchodilators (long-acting muscarinic antagonists [LAMAs] and/or long-acting $\beta_{2}$-agonists [LABAs]) alone or in combination with an inhaled corticosteroid (ICS). ${ }^{2}$ For symptomatic patients with moderate-to-very severe COPD, with or without a history of exacerbations, several Phase III studies have demonstrated the benefits of triple ICS/ LAMA/LABA combinations compared with dual LAMA/ LABA and ICS/LABA therapies on lung function, symptoms, and COPD exacerbations. ${ }^{3-6}$ Nevertheless, it is important to consider the long-term safety of these treatments to determine their overall risk/benefit profile, as the prolonged use of ICS in COPD has been associated with the potential for adverse events (AEs), including pneumonia and cataracts. ${ }^{2}$

To date, three triple fixed-dose combination ICS/LAMA/ LABA therapies have been developed, ${ }^{7}$ including budesonide/glycopyrrolate/formoterol fumarate metered dose inhaler (BGF MDI), which is formulated using co-suspension delivery technology. ${ }^{8}$ The efficacy and safety of BGF MDI were assessed in the pivotal Phase III KRONOS study, which found that triple therapy had benefits on lung function, symptoms, and exacerbations versus dual therapy with glycopyrrolate/formoterol fumarate (GFF) MDI or budesonide/ formoterol fumarate (BFF) $\mathrm{MDI}^{3}$ In addition, the safety profile of BGF MDI was comparable to that of both dual therapies. However, as ethnic and regional factors can result in differences in drug response, ${ }^{9}$ it is important to assess the long-term safety and efficacy of these therapies in a variety of populations. KRONOS included 416 patients enrolled in Japan, and efficacy and safety findings in the Japanese subgroup were generally comparable to the overall population. ${ }^{10}$ To further characterize the risk/benefit profile of triple therapy in Japanese patients, we evaluated the long-term safety and efficacy of BGF MDI over 52 weeks in patients from Japan with moderate-to-very severe COPD who participated in a 28 -week extension of the 24 -week KRONOS study.

\section{Materials and Methods \\ Study Design}

KRONOS (NCT02497001) was a Phase III, double-blind, multicenter parallel-group study in which patients were randomized 2:2:1:1 to receive BGF MDI 320/18/9.6 $\mu \mathrm{g}$, GFF MDI 18/9.6 $\mu \mathrm{g}$, BFF MDI 320/9.6 $\mu \mathrm{g}$, or open-label budesonide/formoterol dry powder inhaler (BUD/FORM DPI; Symbicort Turbuhaler ${ }^{\circledR}$ ) 400/12 $\mu$ g, twice-daily for
24 weeks. $^{3}$ The doses of glycopyrrolate and formoterol fumarate (18 $\mu \mathrm{g}$ and $9.6 \mu \mathrm{g}$, respectively) are equivalent to $14.4 \mu \mathrm{g}$ glycopyrronium and $10 \mu \mathrm{g}$ formoterol fumarate dihydrate. BUD/FORM DPI 400/12 $\mu \mathrm{g}$ was administered as two inhalations of $200 / 6 \mu \mathrm{g}$ (metered dose, corresponding to a total daily delivered dose of $320 / 9 \mu \mathrm{g}$ ). During the extension study (NCT03262012), Japanese patients receiving BGF MDI, GFF MDI, BFF MDI, or BUD/FORM DPI in KRONOS continued their randomized treatment for up to 28 additional weeks, resulting in a total of 52 weeks' treatment (Figure 1).

The study was conducted in accordance with Good Clinical Practice, including the Declaration of Helsinki. The protocol and informed consent form were approved by appropriate institutional review boards (full details have been published). ${ }^{3}$ All patients provided written informed consent before screening.

\section{Patients}

Full inclusion/exclusion criteria for KRONOS have been published previously, ${ }^{3}$ and these criteria also applied to the extension study. Briefly, requirements for study entry in KRONOS included an established clinical history of COPD (defined for patients in Japan using Japanese Respiratory Society guidelines ${ }^{11}$ ), a smoking history of $\geq 10$ pack-years, a COPD Assessment Test (CAT) score $\geq 10$ despite receiving two or more inhaled maintenance therapies for at least 6 weeks before screening, and a post-bronchodilator forced expiratory volume in $1 \mathrm{~s}\left(\mathrm{FEV}_{1}\right) \geq 25 \%$ and $<80 \%$, according to predicted normal values using reference norms for Japan. $^{11,12}$ There was no requirement for a history of COPD exacerbations in the year prior to study entry. Patients with a current diagnosis of asthma were excluded.

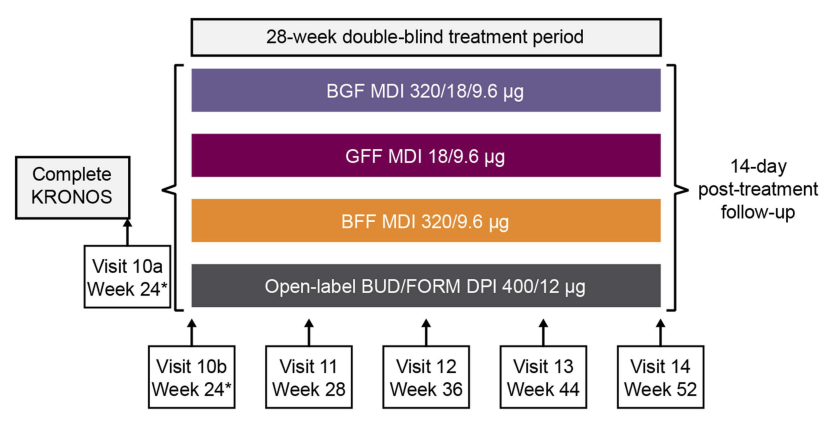

Figure I Study design.

Notes: *Visits 10a and $10 \mathrm{~b}$ were conducted on the same day. Visit $10 \mathrm{~b}$ began immediately following completion of Visit 10a procedures.

Abbreviations: BFF, budesonide/formoterol fumarate; BGF, budesonide/glycopyrrolate/formoterol fumarate; BUD/FORM DPI, budesonide/formoterol fumarate dry powder inhaler; GFF, glycopyrrolate/formoterol fumarate; MDI, metered dose inhaler. 
In addition, for inclusion in the 28-week extension study, patients had to give informed consent to participate, and complete the treatment phase of KRONOS compliant with study procedures and study drug dosing.

\section{Assessments}

The primary objective was to evaluate the long-term safety and tolerability of BGF MDI, GFF MDI, BFF MDI, and BUD/ FORM DPI in Japanese patients with moderate-to-very severe COPD. Safety assessments included AE monitoring, 12-lead electrocardiograms, clinical laboratory testing, and vital sign measurements. AEs of special interest (AESIs) included pneumonia, paradoxical bronchospasm, and several classes of AEs identified based on pharmacologically predictable effects, including: cardiovascular effects, ocular disorders, urinary retention, gastrointestinal disorders, and anticholinergic effects (LAMAs); cardiovascular effects, tremor, hyperglycemia, and hypokalemia (LABAs); and local (e.g., candidiasis and voice effects) and systemic (e.g., bone and skin effects, diabetes control, ocular and taste effects, adrenal suppression) steroid class effects, and lung infection (ICS). The relationship of AEs to the study treatment was assessed by the investigators after careful consideration, and according to the guidelines outlined in the Supplementary Materials.

An external clinical endpoint committee (CEC) that was initiated in KRONOS continued to adjudicate pneumonia, major adverse cardiovascular events (MACE), and causes of death for the extension phase of the study to ensure appropriate pre-defined and clinically consistent criteria were met. An external data monitoring committee monitored the safety data at predetermined intervals.

Efficacy assessments included change from baseline in morning pre-dose trough $\mathrm{FEV}_{1}$ over 52 weeks, rate of moderate or severe COPD exacerbations (as defined in $\mathrm{KRONOS}^{3}$ ), change from baseline in average daily rescue medication use, and change from baseline in the Evaluating Respiratory Symptoms (E-RS) in COPD total score, as well as three subscale scores (breathlessness, cough and sputum, and chest symptoms) over 52 weeks. Baseline assessments were conducted as part of enrollment in the KRONOS study, either during screening (for rescue medication use and E-RS scores) or at randomization (for $\mathrm{FEV}_{1}$ ).

\section{Statistical Analysis}

A sample size of 324 Japanese patients from KRONOS (BGF MDI, n=108; GFF MDI, n=108; BFF MDI, $\mathrm{n}=54$ : BUD/FORM DPI, $n=54$ ) was selected to provide approximately 100 completing patients in the BGF MDI and GFF
MDI arms. The Japanese safety and modified intent-to-treat (mITT) populations were subgroups of the global KRONOS safety and mITT populations that included the patients enrolled at sites in Japan, regardless of participation in the extension study. Patients who did not receive any study drug were excluded from the Japanese safety population. Hypothesis testing was not performed for any safety analyses. Variables were summarized with descriptive statistics, frequency counts, and percentages, as appropriate.

Efficacy assessments were considered to be exploratory as the primary purpose of the extension study was to assess safety. For the efficacy assessments, the estimand of interest was the efficacy estimand (the effect of the randomized treatment assuming continuation of randomized treatments for the duration of the study, regardless of actual adherence to study medication), for which analyses were conducted in the Japanese mITT population, using data obtained prior to patients discontinuing from treatment. The change from baseline in morning pre-dose trough $\mathrm{FEV}_{1}$ over 52 weeks was analyzed using a repeated measures linear mixed model including treatment, visit, treatment-by-visit interaction, ICS use at screening, baseline trough $\mathrm{FEV}_{1}$, baseline eosinophil count, and percent reversibility to albuterol. Rate of moderate or severe COPD exacerbations, change from baseline in average daily rescue medication use, and change from baseline in E-RS scores were summarized descriptively.

\section{Results \\ Study Population}

A total of 416 Japanese patients were randomized and treated in KRONOS $(21.9 \%$ of the global study population; Figure 2). These patients were included in the Japanese KRONOS safety and mITT populations. Of these, 377 patients $(90.6 \%)$ completed 24 weeks of treatment in KRONOS and were eligible to continue in the extension study, and 347 patients (83.4\%) consented to participate and were treated for up to an additional 28 weeks. Full demographic characteristics of the Japanese safety population have been published. ${ }^{10}$ In brief, the majority of Japanese patients were male $(94.2 \%)$ with a mean age of 69.5 years. Eighty percent of patients had not experienced a COPD exacerbation in the previous year, and only $31.5 \%$ were receiving an ICS at study entry. In general, demographics were balanced across treatment groups in the Japanese population; however, the proportion of patients who had experienced at least one exacerbation in the previous year 


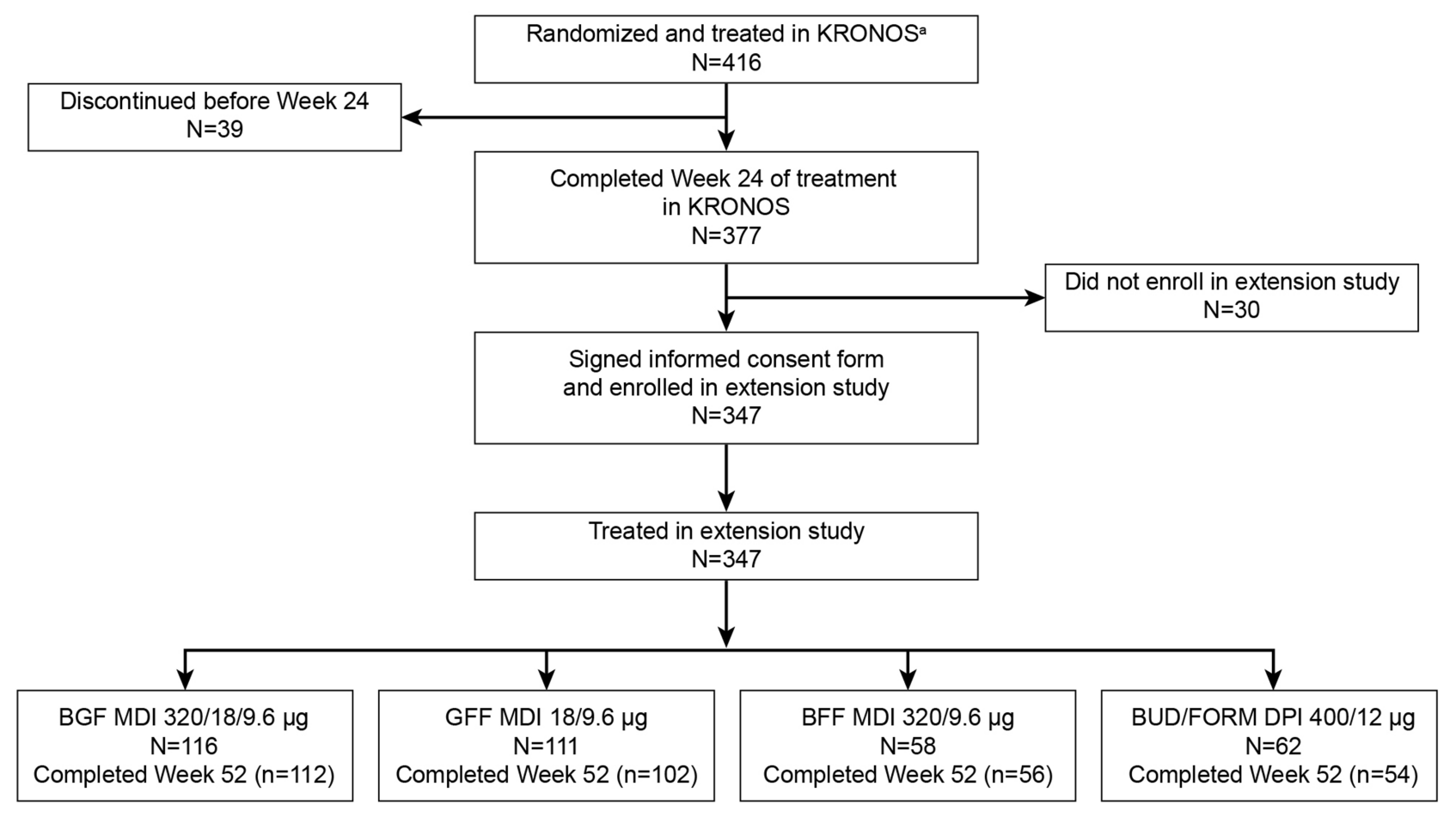

Figure 2 Patient disposition.

Notes: ${ }^{\mathrm{a}}$ All 416 Japanese patients who were randomized and treated in KRONOS were included in the Japanese mITT and safety populations, regardless of participation in the extension study.

Abbreviations: BFF, budesonide/formoterol fumarate; BGF, budesonide/glycopyrrolate/formoterol fumarate; BUD/FORM DPI, budesonide/formoterol fumarate dry powder inhaler; GFF, glycopyrrolate/formoterol fumarate; MDI, metered dose inhaler; mITT, modified intent-to-treat.

was larger in the BGF MDI group (25.9\%) compared to the dual therapy groups (range: 15.9-17.4\%). The median study drug exposure was 364.0 days in all four treatment groups; overall, $79.1 \%$ of patients had $\geq 48$ weeks of exposure.

\section{Safety}

Over 52 weeks, the majority of patients (82.7\%) experienced at least 1 treatment-emergent AE (TEAE); most were mild or moderate in intensity and were not considered by the investigator to be treatment-related (Table 1). The incidence of TEAEs considered related to study treatment was similar in the BGF MDI and BFF MDI groups (24.5\% and $22.9 \%$, respectively) and lower in the GFF MDI and BUD/FORM DPI groups (11.6\% and $13.0 \%$, respectively; Table 1$)$. The incidence of serious TEAEs ranged from $15.1 \%$ to $21.7 \%$ across treatment groups and was highest in the GFF MDI group (Table 1). The most commonly reported TEAEs overall were nasopharyngitis $(32.2 \%)$, bronchitis $(9.9 \%)$, and muscle spasms (7.5\%; Table 2). In general, the profile and frequency of TEAEs were similar across treatment groups. Dysphonia and candidiasis, pharmacologically expected local steroid effects, occurred more frequently in the three ICS-containing treatment groups. Among the preferred terms identified as AESIs, only bronchitis, pneumonia, dysphonia, and candidiasis occurred in $\geq 3 \%$ of patients in any treatment group (Table 2).

To evaluate the association between treatment exposure and TEAE incidence, we assessed TEAEs over time across 12-week intervals. Although the incidence of individual TEAEs fluctuated within and across treatment groups over time, there was no evidence that the frequency of individual TEAEs increased based on the duration of exposure (Table 3).

Four patients experienced a CEC-confirmed MACE: one cardiovascular death in both the BGF MDI and GFF MDI groups, and two non-fatal myocardial infarctions in the BUD/FORM DPI group. None were considered by the investigator to be related to study treatment. The percentage of patients with CEC-confirmed pneumonia events ranged from $2.9 \%$ to $9.4 \%$ across the treatment groups, with the highest percentage in the BGF MDI group (Table 1).

While the incidence of confirmed pneumonia during the first 24 weeks was highest in the BGF MDI group compared with the dual therapy groups, this pattern did not 
Table I Summary of Adverse Events (Japanese Safety Population)

\begin{tabular}{|c|c|c|c|c|}
\hline TEAEs, $\mathbf{n}(\%)$ & $\begin{array}{l}\text { BGF MDI } \\
320 / 18 / 9.6 \mu g \text { N=139 }\end{array}$ & $\begin{array}{l}\text { GFF MDI } \\
\text { I 8/9.6 } \mu \mathrm{g} N=\text { | } 38\end{array}$ & $\begin{array}{l}\text { BFF MDI } \\
320 / 9.6 \mu g \quad N=70\end{array}$ & $\begin{array}{l}\text { BUD/FORM DPI } \\
400 / 12 \mu g \quad N=69\end{array}$ \\
\hline Patients with $\geq I \mathrm{TEAE}$ & IIS (82.7) & II4 (82.6) & $58(82.9)$ & $57(82.6)$ \\
\hline Mild & $66(47.5)$ & $60(43.5)$ & $36(51.4)$ & $31(44.9)$ \\
\hline Moderate & $34(24.5)$ & $33(23.9)$ & $17(24.3)$ & $17(24.6)$ \\
\hline Severe & $15(10.8)$ & $21(15.2)$ & $5(7.1)$ & $9(13.0)$ \\
\hline Patients with TEAEs related ${ }^{\mathrm{a}}$ to study treatment & $34(24.5)$ & $16(11.6)$ & $16(22.9)$ & $9(13.0)$ \\
\hline Patients with TEAEs that led to early discontinuation & $10(7.2)$ & $12(8.7)$ & $4(5.7)$ & $6(8.7)$ \\
\hline Patients with serious TEAEs & $21(15.1)$ & $30(21.7)$ & II (I5.7) & $14(20.3)$ \\
\hline Patients with serious TEAEs related ${ }^{a}$ to study treatment & $3(2.2)$ & $7(5.1)$ & $2(2.9)$ & $3(4.3)$ \\
\hline Patients with confirmed ${ }^{\mathrm{b}} \mathrm{MACE}$ & $\mathrm{I}(0.7)$ & I (0.7) & 0 & $2(2.9)$ \\
\hline Patients with confirmed ${ }^{\mathrm{b}}$ pneumonia & $13(9.4)$ & $5(3.6)$ & $4(5.7)$ & $2(2.9)$ \\
\hline At or before 24 weeks & $7(5.0)$ & I (0.7) & 0 & 0 \\
\hline During weeks $25-52$ & $6(4.3)$ & $4(2.9)$ & $4(5.7)$ & $2(2.9)$ \\
\hline Deaths (all causes) & $3(2.2)$ & I (0.7) & $\mathrm{I}(\mathrm{I} .4)$ & I ( 1.4$)$ \\
\hline
\end{tabular}

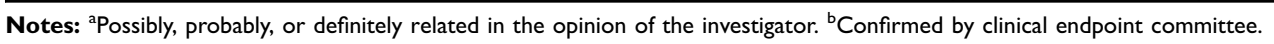

Abbreviations: BFF, budesonide/formoterol fumarate; BGF, budesonide/glycopyrrolate/formoterol fumarate; BUD/FORM DPI, budesonide/formoterol fumarate dry powder inhaler; GFF, glycopyrrolate/formoterol fumarate; MACE, major adverse cardiovascular events; MDI, metered dose inhaler; TEAE, treatment-emergent adverse event.

Table 2 Adverse Events Occurring in $\geq 3 \%$ of Patients in Any Treatment Group (Japanese Safety Population)

\begin{tabular}{|c|c|c|c|c|}
\hline Preferred Term, n (\%) & $\begin{array}{l}\text { BGF MDI } \\
320 / 18 / 9.6 \mu g \mathrm{~N}=139\end{array}$ & $\begin{array}{l}\text { GFF MDI } \\
\text { | } 8 / 9.6 \mu g ~ N=138\end{array}$ & $\begin{array}{l}\text { BFF MDI } \\
320 / 9.6 \mu g ~ N=70\end{array}$ & $\begin{array}{l}\text { BUD/FORM DPI } \\
400 / / 2 \mu \mathrm{g} N=69\end{array}$ \\
\hline Nasopharyngitis & $45(32.4)$ & $43(31.2)$ & $22(31.4)$ & $24(34.8)$ \\
\hline Bronchitis & $15(10.8)$ & II (8.0) & $8(11.4)$ & $7(10.1)$ \\
\hline Muscle spasms & $16(11.5)$ & $6(4.3)$ & $6(8.6)$ & $3(4.3)$ \\
\hline Pneumonia $^{\mathrm{a}}$ & $14(10.1)$ & $5(3.6)$ & $4(5.7)$ & $4(5.8)$ \\
\hline Dysphonia & $10(7.2)$ & I (0.7) & $9(12.9)$ & $3(4.3)$ \\
\hline $\mathrm{COPD}^{\mathrm{b}}$ & $7(5.0)$ & II (8.0) & $2(2.9)$ & $2(2.9)$ \\
\hline Upper respiratory tract infection & $10(7.2)$ & $8(5.8)$ & $\mathrm{I}(\mathrm{I} .4)$ & $2(2.9)$ \\
\hline Influenza & $5(3.6)$ & $7(5.1)$ & $3(4.3)$ & $6(8.7)$ \\
\hline Upper respiratory tract inflammation & $5(3.6)$ & $7(5.1)$ & $5(7.1)$ & 0 \\
\hline Constipation & $6(4.3)$ & $5(3.6)$ & $4(5.7)$ & $2(2.9)$ \\
\hline Pharyngitis & $3(2.2)$ & $5(3.6)$ & $3(4.3)$ & $2(2.9)$ \\
\hline Eczema & I (0.7) & $3(2.2)$ & $4(5.7)$ & $4(5.8)$ \\
\hline Oral candidiasis & $5(3.6)$ & 0 & $3(4.3)$ & $3(4.3)$ \\
\hline Pyrexia & $3(2.2)$ & $6(4.3)$ & 0 & 0 \\
\hline Oropharyngeal pain & $5(3.6)$ & I (0.7) & I (I.4) & I (I.4) \\
\hline Contusion & $5(3.6)$ & I (0.7) & 0 & I (I.4) \\
\hline
\end{tabular}

Notes: a'Spontaneously reported as AEs; not confirmed by clinical endpoint committee. ${ }^{b}$ Worsening of COPD.

Abbreviations: AE, adverse event; BFF, budesonide/formoterol fumarate; BGF, budesonide/glycopyrrolate/formoterol fumarate; BUD/FORM DPI, budesonide/formoterol fumarate dry powder inhaler; GFF, glycopyrrolate/formoterol fumarate; MDI, metered dose inhaler.

continue in the 28-week extension phase of the study. In the BGF MDI group, the incidence of confirmed pneumonia was similar in the first 24 weeks $(5.0 \%)$ and after 24 weeks $(4.3 \%)$, while in the other three treatment groups incidence was higher after 24 weeks (range: 2.9-5.7\%) compared with the first 24 weeks (range: $0-0.7 \%$; Table 1). Overall, out of 24 confirmed pneumonia events, only five were considered possibly related to study treatment $(n=2$ in the BGF MDI and GFF MDI groups, and $n=1$ in the BUD/FORM DPI group). 


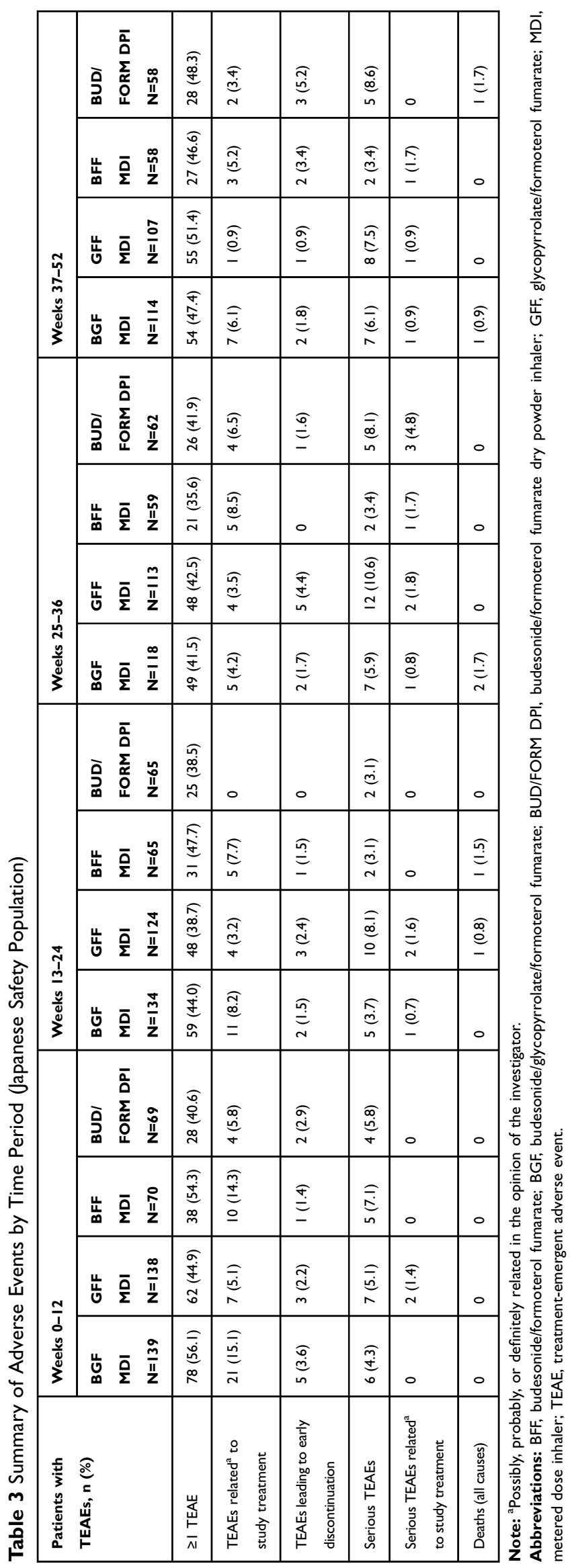


Six deaths were reported during the study, none of which were considered to be treatment-related by the investigator. Three deaths occurred in the BGF MDI group (resulting from a traffic accident, pneumonia with disseminated intravascular coagulation, and lethal arrhythmia). The investigator considered the fatal pneumonia to be a complication of the anticancer treatment that the patient was receiving for colon cancer, and the death due to lethal arrhythmia occurred in a 73-year-old male who suffered a cardiopulmonary arrest after returning home from field work and died in hospital four days later. One death was reported in each of the GFF MDI, BFF MDI, and BUD/FORM DPI groups (resulting from cardio-respiratory arrest, squamous cell carcinoma, and lung adenocarcinoma, respectively).

No clinically meaningful trends were observed in electrocardiogram findings, laboratory parameters, or vital signs over time in any of the treatment groups.

\section{Efficacy}

Treatment with BGF MDI resulted in a larger mean change from baseline in morning pre-dose trough $\mathrm{FEV}_{1}$ (least squares mean [LSM] $117 \mathrm{~mL}$ ) compared with dual therapies (LSM range: $54-85 \mathrm{~mL}$; Supplementary Table 1 ), with the trend of greater improvements in the BGF MDI group maintained over the 52-week treatment period (Figure 3). At baseline, Japanese patients reported low use of rescue medication, ranging from 0.5 to 0.8 puffs of albuterol/day across treatment groups. Correspondingly, mean changes from baseline in daily rescue medication use over 52 weeks were also small and similar for all treatment groups (range: 0 to -0.3 puffs/ day; Supplementary Table 1). The percentage of patients experiencing a moderate or severe COPD exacerbation over 52 weeks ranged from $20.0 \%$ to $31.9 \%$ and was highest in the GFF MDI group (Supplementary Table 1). Rates of moderate or severe exacerbations were comparable across the three ICS-containing treatments (range: $0.26-0.37$ per year) and highest for GFF MDI (0.81 per year; Supplementary Table 1). Changes from baseline in E-RS total score over 52 weeks ranged from -0.2 (GFF MDI) to -0.9 (BGF MDI; Supplementary Table 1). Improvements in RS-breathlessness score were also greater for BGF MDI compared with dual therapies, while changes in RS-cough and sputum and RSchest symptoms scores were similar across treatment groups (Supplementary Table 1).

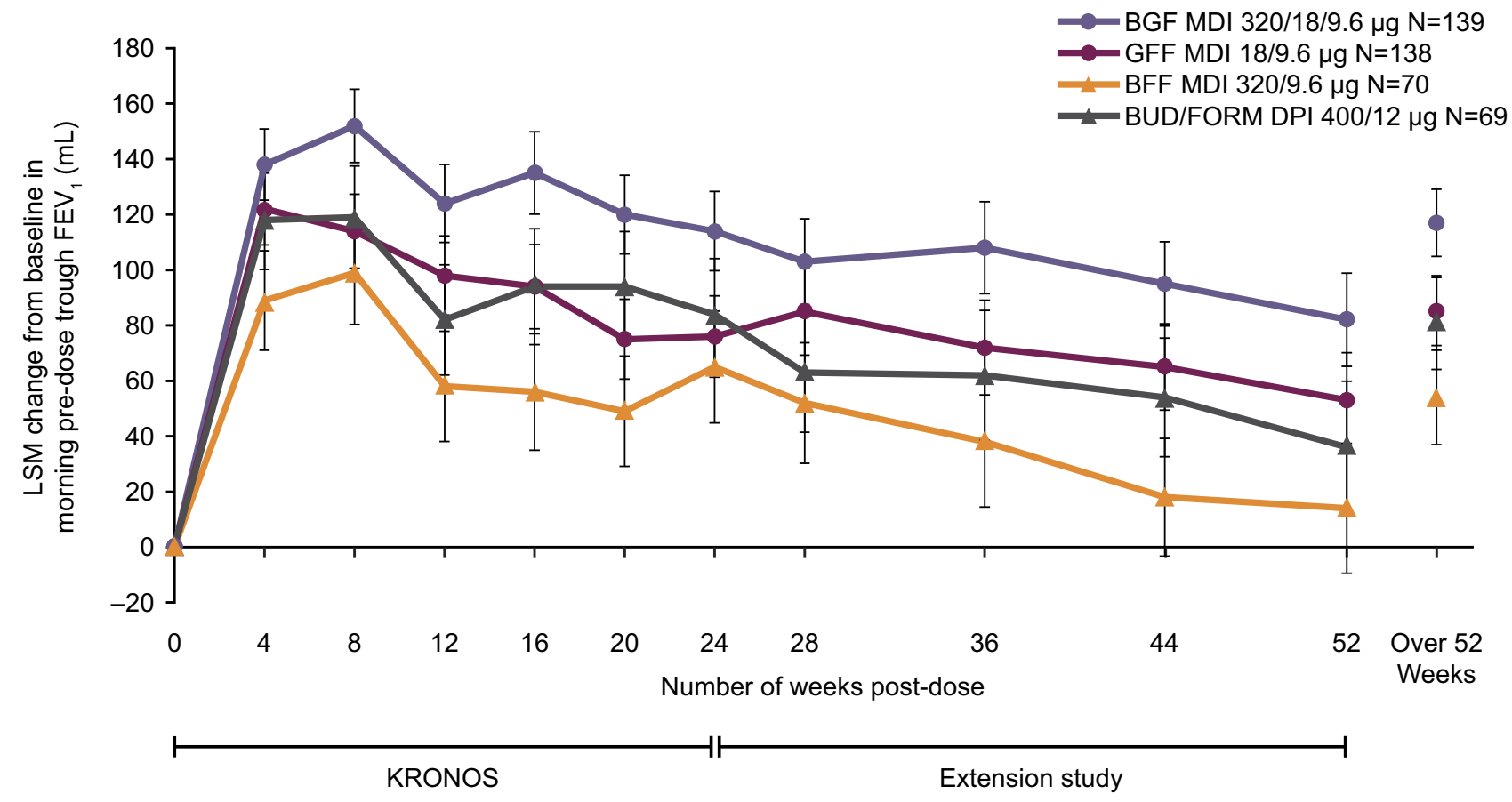

Figure 3 Change from baseline in morning pre-dose trough FEV , over 52 weeks (efficacy estimand; Japanese mITT population). Note: Error bars represent the standard error.

Abbreviations: BFF, budesonide/formoterol fumarate; BGF, budesonide/glycopyrrolate/formoterol fumarate; BUD/FORM DPI, budesonide/formoterol fumarate dry powder inhaler; $\mathrm{FEV}_{1}$, forced expiratory volume in I s; GFF, glycopyrrolate/formoterol fumarate; LSM, least squares mean; MDI, metered dose inhaler; mITT, modified intent-to-treat. 


\section{Discussion}

This 28-week extension study of the KRONOS trial assessed the long-term safety and efficacy of the ICS/LAMA/LABA triple therapy, BGF MDI, over 52 total weeks of treatment in Japanese patients with moderate-to-very severe COPD. BGF MDI was generally well tolerated in Japanese patients, with a safety profile broadly similar to that of the corresponding dual LAMA/LABA and ICS/LABA therapies.

Overall, the incidence of TEAEs was consistent across treatment groups (range: $82.6-82.9 \%$ ), and the majority of reported TEAEs were of mild or moderate intensity. Although the frequency of individual TEAEs fluctuated within and across treatment groups over time, there was no evidence that the profile or frequency of individual TEAEs increased based on the duration of exposure, suggesting no cumulative adverse effects of treatment over time.

A previous history of moderate or severe exacerbations may be a risk factor for the development of pneumonia in patients with COPD. ${ }^{13}$ It should be noted that $25.9 \%$ of patients in the BGF MDI group had experienced an exacerbation in the year prior to enrollment, which was higher than in the dual therapy groups (range: 15.9-17.4\%). In this regard, 5 of the 7 patients in the BGF MDI group with a confirmed pneumonia event in the first 24 weeks of the study had experienced an exacerbation in the year prior to study entry, whereas no patients in the other three treatment groups who developed pneumonia over the 52-week study reported a prior history of exacerbation. This could potentially account for the imbalance of pneumonia in the BGF MDI group in the first 24 weeks of the study, as no patients who developed pneumonia after 24 weeks had a risk factor of a prior exacerbation, and the incidence across groups was comparable in the second half of the study.

Over 52 weeks, pneumonia was confirmed in $2.9 \%$ to $9.4 \%$ of patients across the treatment groups, with the highest percentage in the BGF MDI group. However, only 2 of the 13 confirmed events in the BGF MDI group were considered by the investigator to be related to study treatment. As the incidence of pneumonia was lower in the BFF MDI and BUD/FORM DPI groups relative to the BGF MDI group, this finding does not appear to represent an effect of budesonide treatment. The number of patients with confirmed pneumonia was similar in the first 24 weeks $(n=7)$ and after 24 weeks $(n=6)$ in the BGF MDI group, indicating that pneumonia incidence did not increase with long-term use. In contrast, in the LAMA/LABA and ICS/LABA groups, the incidence of confirmed pneumonia was higher during the 28-week extension phase (range: 2.9-5.7\%) compared with the first 24 weeks (range: 0-0.7\%). Hence, the higher incidence of confirmed pneumonia in the BGF MDI group compared to the other treatment arms was primarily driven by events that occurred in the first 24 weeks of the study, and incidence was generally balanced across treatment groups after Week 24.

The overall incidence of confirmed pneumonia over 52 weeks was higher in Japanese patients (5.8\%) compared with US patients $(2.4 \%)$ who participated in a similar KRONOS extension study. ${ }^{14}$ Other multinational clinical studies in patients with COPD have also reported a higher incidence of pneumonia in Japanese and Asian patients relative to the full study population. ${ }^{15,16}$ This may be partly due to the older average age of the Japanese population (69.5 vs 62.8 years in the US extension study) and the higher frequency of diagnostic X-ray use in Japan versus the USA. ${ }^{17}$ Overall, these factors suggest that while the incidence of pneumonia was higher overall in Japanese patients compared to a previous 52 -week study conducted in the USA, ${ }^{14}$ with the highest incidence observed in the BGF MDI group, these findings may have been influenced by regional effects and an imbalance in risk factors across treatment groups (e.g., prior exacerbation history), and were likely not treatment-related.

Overall, results for efficacy endpoints with BGF MDI were generally consistent with the findings in the Japanese and global populations of KRONOS, ${ }^{3,10}$ with greater improvement in lung function versus GFF MDI and BFF MDI, and reduction in symptoms and exacerbations versus GFF MDI. While efficacy analyses were considered exploratory due to the relatively small sample size of this safety extension study, the findings demonstrated that the treatment effects observed at 24 weeks ${ }^{10}$ were sustained over 52 weeks, and therefore support the long-term efficacy of BGF MDI.

\section{Conclusions}

This 52-week study found that the safety profile of BGF MDI was generally comparable to dual LAMA/LABA and ICS/LABA therapies in Japanese patients with moderate-tovery severe COPD. All treatments were well tolerated, and there was no evidence that the profile or frequency of individual TEAEs differed based on the duration of exposure within or across treatment groups. Safety and efficacy results over 52 weeks were generally in agreement with findings from the global population and the Japanese subgroup of KRONOS over 24 weeks, ${ }^{3,10}$ and support the long-term tolerability of BGF MDI in Japanese patients with moderateto-very severe COPD. 


\section{Abbreviations}

AE, adverse event; AESI, adverse event of special interest; BFF, budesonide/formoterol fumarate; BGF, budesonide/glycopyrrolate/formoterol fumarate; BUD/FORM DPI, budesonide/formoterol dry powder inhaler; CEC, clinical endpoint committee; E-RS, Evaluating Respiratory Symptoms; FEV ${ }_{1}$, forced expiratory volume in $1 \mathrm{~s}$; GFF, glycopyrrolate/formoterol fumarate; ICS, inhaled corticosteroid; LABA, longacting $\beta_{2}$-agonist; LAMA, long-acting muscarinic antagonist; LSM, least squares mean; MACE, major adverse cardiovascular events; MDI, metered dose inhaler; mITT, modified intent-to-treat; TEAE, treatment-emergent adverse event.

\section{Ethics Approval and Informed Consent}

Patients provided written informed consent prior to screening, and the study was conducted in accordance with Good Clinical Practice, including the Declaration of Helsinki and the International Council for Harmonisation. The protocol was approved by local institutional review boards (names and protocol numbers have been previously published). ${ }^{3}$ The names of all institutional review boards can be found in Supplementary Table 2 .

\section{Data Availability}

Data underlying the findings described in this manuscript may be obtained in accordance with AstraZeneca's data sharing policy described at: https://astrazenecagrouptrials. pharmacm.com/ST/Submission/Disclosure.

\section{Acknowledgments}

The authors would like to thank all the patients and their families and the team of investigators, research nurses, and operations staff involved in these studies. Medical writing support, under the direction of the authors, was provided by Julia King, $\mathrm{PhD}$, of $\mathrm{CMC}$ Connect, a division of McCann Health Medical Communications Ltd, Glasgow, UK and was funded by AstraZeneca, Gaithersburg, USA, in accordance with Good Publication Practice (GPP3) guidelines. ${ }^{18}$

\section{Author Contributions}

$\mathrm{HO}, \mathrm{MT}, \mathrm{EB}, \mathrm{SB}, \mathrm{KdA}, \mathrm{PD}$, and CR contributed to the conception or design of the study; MI, YF, YI, OH, GTF, KFR, HO, MT, EB, KdA, and MA participated in the acquisition of reported data; $\mathrm{NH}$ and SB contributed to the statistical analysis of the data. All authors participated in the analysis and interpretation of data reported. All authors reviewed or critically revised the manuscript, provided the final approval of the version to be published, and agreed to be accountable for all aspects of the work.

\section{Funding}

The KRONOS study was supported by AstraZeneca.

\section{Disclosure}

MI reports personal fees from AstraZeneca, during the conduct of the study; and personal fees from Kyorin, Nippon Boehringer Ingelheim, and Novartis Pharma, outside of the submitted work. YI reports personal fees from GlaxoSmithKline, Nippon Boehringer Ingelheim, Nobelpharma, and Shionogi \& Co. Ltd, outside of the submitted work. $\mathrm{OH}$ reports personal fees from AstraZeneca, Nippon Boehringer Ingelheim, and Novartis Pharma; and research funding from AstraZeneca, Daiichi Sankyo, GlaxoSmithKline, Nippon Boehringer Ingelheim, and Novartis Pharma. GTF reports grants, personal fees, and nonfinancial support from AstraZeneca during the conduct of the study; grants, personal fees, and non-financial support from AstraZeneca, Boehringer Ingelheim, Novartis, Pearl a member of the AstraZeneca Group, and Sunovion; grants and personal fees from Theravance; and personal fees from Circassia, GlaxoSmithKline, Innoviva, Mylan, and Verona, outside of the submitted work. KFR reports personal fees from AstraZeneca, Berlin-Chemie, Boehringer Ingelheim, Chiesi Pharmaceuticals, InterMune, Novartis, Sanofi, and Teva; and grants from the Ministry of Education and Science, Germany, outside of the submitted work. NH, HO, and MT are employees of AstraZeneca K.K., Japan. EB, SB, $\mathrm{MA}, \mathrm{CR}$, and PD are employees of AstraZeneca. KdA is a former employee of AstraZeneca. The authors report no other conflicts of interest in this work.

\section{References}

1. Global Initiative for Chronic Obstructive Lung Disease Japan. Statistical data on COPD. 2017. Available from: http://www.gold-jac. jp/copd facts in japan/. Accessed November 14, 2018.

2. Global Initiative for Chronic Obstructive Lung Disease. 2019 Report: global strategy for the diagnosis, management and prevention of COPD 2019. Available from: https://goldcopd.org. Accessed June 28, 2019.

3. Ferguson GT, Rabe KF, Martinez FJ, et al. Triple therapy with budesonide/ glycopyrrolate/formoterol fumarate with co-suspension delivery technology versus dual therapies in chronic obstructive pulmonary disease (KRONOS): a double-blind, parallel-group, multicentre, phase 3 randomised controlled trial. Lancet Respir Med. 2018;6(10):747-758. doi:10.1016/S2213-2600(18)30327-8 
4. Papi A, Vestbo J, Fabbri L, et al. Extrafine inhaled triple therapy versus dual bronchodilator therapy in chronic obstructive pulmonary disease (TRIBUTE): a double-blind, parallel group, randomised controlled trial. Lancet. 2018;391(10125):1076-1084. doi:10.1016/ S0140-6736(18)30206-X

5. Lipson DA, Barnhart F, Brealey N, et al. Once-daily single-inhaler triple versus dual therapy in patients with COPD. $N$ Engl J Med. 2018;378(18):1671-1680. doi:10.1056/NEJMoa1713901

6. Singh D, Papi A, Corradi M, et al. Single inhaler triple therapy versus inhaled corticosteroid plus long-acting $\beta_{2}$-agonist therapy for chronic obstructive pulmonary disease (TRILOGY): a double-blind, parallel group, randomised controlled trial. Lancet. 2016;388(10048):963-973. doi:10.1016/S0140-6736(16)31354-X

7. Lipworth B, Kuo CR, Jabbal S. Current appraisal of single inhaler triple therapy in COPD. Int J Chron Obstruct Pulmon Dis. 2018;13:3003-3009. doi:10.2147/COPD

8. Ferguson GT, Hickey AJ, Dwivedi S. Co-suspension delivery technology in pressurized metered dose inhalers for multi-drug dosing in the treatment of respiratory diseases. Respir Med. 2018;134:16-23. doi:10.1016/j.rmed.2017.09.012

9. Yasuda SU, Zhang L, Huang S-M. The role of ethnicity in variability in response to drugs: focus on clinical pharmacology studies. Clin Pharmacol Ther. 2008;84(3):417-423. doi:10.1038/ clpt.2008.141

10. Ichinose M, Fukushima Y, Inoue Y, et al. Efficacy and safety of budesonide/glycopyrrolate/formoterol fumarate metered dose inhaler formulated using co-suspension delivery technology in Japanese patients with COPD: a subgroup analysis of the KRONOS study. Int J Chron Obstruct Pulmon Dis. 2019;14:2979-2991
11. The Japanese Respiratory Society. Guidelines for the Diagnosis and Treatment of COPD. 4th Edition. (in Japanese); 2013. Available from http://www.jrs.or.jp/. Accessed April 9, 2019.

12. Sasaki H, Nakamura M, Kida K, et al. Reference values for spirogram and blood gas analysis in Japanese adults. J Jpn Respir Soc. 2001;39(5):S1-S17.

13. Müllerova H, Chigbo C, Hagan GW, et al. The natural history of community-acquired pneumonia in COPD patients: a population database analysis. Respir Med. 2012;106(8):1124-1133. doi:10.1016/j. rmed.2012.04.008

14. Kerwin EM, Ferguson GT, Mo M, DeAngelis K, Dorinsky P. Bone mineral density and ocular safety after 52 weeks' treatment with budesonide/glycopyrrolate/formoterol fumarate metered dose inhaler (BGF MDI) using co-suspension delivery technology in COPD. Am J Respir Crit Care Med. 2019;199(Suppl.):A3319.

15. Ichinose M, Nishimura M, Akimoto M, et al. Tiotropium/olodaterol versus tiotropium in Japanese patients with COPD: results from the DYNAGITO study. Int J Chron Obstruct Pulmon Dis. 2018;13: 2147-2156. doi:10.2147/COPD

16. Wedzicha JA, Zhong N, Ichinose M, et al. Indacaterol/glycopyrronium versus salmeterol/fluticasone in Asian patients with COPD at a high risk of exacerbations: results from the FLAME study. Int J Chron Obstruct Pulmon Dis. 2017;12:339-349. doi:10.2147/COPD

17. Berrington de González A, Darby S. Risk of cancer from diagnostic X-rays: estimates for the UK and 14 other countries. Lancet. 2004;363(9406):345-351. doi:10.1016/S0140-6736(04)15433-0

18. Battisti WP, Wager E, Baltzer L, et al. Good publication practice for communicating company-sponsored medical research: GPP3. Ann Intern Med. 2015;163(6):461-464. doi:10.7326/M15-0288

\section{Publish your work in this journal}

The International Journal of COPD is an international, peer-reviewed journal of therapeutics and pharmacology focusing on concise rapid reporting of clinical studies and reviews in COPD. Special focus is given to the pathophysiological processes underlying the disease, intervention programs, patient focused education, and self management protocols. This journal is indexed on PubMed Central, MedLine and CAS. The manuscript management system is completely online and includes a very quick and fair peer-review system, which is all easy to use. Visit http://www.dovepress.com/testimonials.php to read real quotes from published authors. 